Euskal ikerketen aldizkaria | Revue d'études basques |

Revista de estudios vascos | Basque studies review

$9 \mid 2004$

Numéro IX

\title{
Eguiatéguy, lecteur de Ioannes Etcheberri de Ciboure
}

\section{Aurélie Arcocha-Scarcia}

\section{OpenEdition}

\section{Journals}

Édition électronique

URL : http://journals.openedition.org/lapurdum/785

DOI : 10.4000/lapurdum.785

ISSN : 1965-0655

\section{Éditeur}

IKER

Édition imprimée

Date de publication : 1 novembre 2004

Pagination : 49-66

ISBN : 2-68781-376-X

ISSN : $1273-3830$

Référence électronique

Aurélie Arcocha-Scarcia, «Eguiatéguy, lecteur de loannes Etcheberri de Ciboure », Lapurdum [En ligne], 9 | 2004, mis en ligne le 01 mars 2009, consulté le 01 mai 2019. URL : http://journals.openedition.org/ lapurdum/785 ; DOI : 10.4000/lapurdum.785 


\title{
Eguiatéguy, lecteur de Ioannes Etcheberri de Ciboure
}

\author{
Aurélie ARCOCHA-SCARCIA \\ Université Michel de Montaigne Bordeaux 3 \\ IKER-UMR 5478 du CNRS
}

Le texte Mundiren Berritzias Mosdé Etcheberri-ren Gogomenac ${ }^{1}$ [Les pensées de Monsieur Etcheberri sur le Renouvellement du Monde], objet principal de cet article, se trouve dans le Fonds Celtique et Basque $\mathrm{n}^{\circ} 154$ de la Bibliothèque Nationale de France (FCB 154) parmi divers écrits de Joseph Eguiatéguy. La seule donnée biographique certaine sur cet auteur est fournie par la mention de son métier Suberoan Erregent, [Régent en Soule] figurant dans ses manuscrits. Avant de nous pencher sur ce texte énigmatique, il est nécessaire de procéder à une brève présentation du corpus éguiatéguien, souvent référencé sans un recours aux manuscrits, avec des informations de seconde, voire de troisième main.

Hormis l'ouvrage d'économie domestique aberatz-tarzun gussien guils bakhoïtza ${ }^{2}$ soit [Clés pour chacune des richesses générales], perdu au cours de la seconde partie du XXe siècle ${ }^{3}$ (Peillen in Egiategi 1983: 13), tous les autres manuscrits d'Eguiatéguy répertoriés à ce jour se trouvent dans ce même fonds de la BNF, sous trois rubriques :

1. FCB 154. Sur le catalogue des manuscrits "celtiques et basques" de la BNF établi par Henri Omont en 1890, l'ensemble conservé sous cette rubrique porte le titre : Sentences et proverbes d'Oybenart. Mais quand on consulte le volume répertorié sous ladite rubrique, on s'aperçoit qu'il porte le titre: Proverbes et Sentences. Il est constitué de trois parties ${ }^{4}$. La première est une adaptation des proverbes bilingues d'Oihenart (1592-1667) d'après, semble-t-il, l'édition de Paris 1657, les deux autres en sont des hypertextes, des imitationes et amplificationes dues à Eguiatéguy. Pour Joseph Eguiatéguy, s'intéresser aux proverbes d'Oihenart, auteur dont il connaît également l'œuvre historique et poétique ${ }^{5}$, et collecter plusieurs centaines de

1 - Pour cette transcription ainsi que pour l'ensemble de celles que je propose tout au long de cet article, j'utilise le code suivant :

[ ] : traduction qui m'incombe ou remarque que j'insère à l'intérieur d'une citation afin de lever une ambiguité. $\bullet()$ : lecture douteuse. $\bullet<>$ : adjonction. $\bullet$ : mot barré. $\cdot(?)$ : illisible.

2 - Lexistence de l'ouvrage est indiquée par Eguiatéguy dans la Préface ou Hitzaoria du FCB 155 f.4.

3 - Il ne se trouve pas, comme on l'a cru, dans le Fonds Celtique \& Basque $n^{\circ} 156$ (FCB 156) de la BNF (Urquizu $2000: 272$ )

4 - Les premières pages du troisième cahier sont manquantes. On peut également observer que le classement par ordre alphabétique y est parfois bouleversé.

5 - Eguiatéguy, admirateur de l'ceuvre de son compatriote souletin "Arlandé Oyhénart", connaît l'existence des deux éditions de Notitia utriusque Vasconiae (Paris $1638 ; 1656$ ) ainsi que celles des proverbes et des poésies ayant paru à Paris (1657) et à Pau (1664?) : "... ainsi que les Notitia utriusque Vasconiae, les Notices des Pays Basques le démontrent ; production veritablement merveilleuse par les belles recherches quelle contient. Il en <est> peu en effet de cette espece, qui jouisse d'une reputation pareille dans la Republique des Lettres ; (...) Il donna pendant sa vie deux Editions de son Excellent ouvrage avec la gloire de la sçavoir épuisée (...)". Il avait laissé quantité de manuscrits qui <pour avoir été jugés illisibles> ont passé à des Etrangers, au grand domage de la Patrie, pour laquelle cette perte est irréparable.

Il subsiste encore de lui un petit Livre imprimé en partie a Paris, et l'autre a Pau, qui traite des proverbes que nous venons de rapporter, on y trouve aussi deux chapitres ; dans le premier il parle de sa jeunesse, et dans 
proverbes, sentences et maximes ${ }^{6}$, fait partie intégrante de son travail de philosophe. Eguiatéguy ira ainsi jusqu'à rassembler plus de 2800 sentences, cas unique dans les lettres basques. Une petite mention bilingue griffonnée à la fin de l'Avertissement nous éclaire sur sa démarche : Eguiatéguy, l'esprit fixé sur l'hypotexte oihenartien, a procédé en se servant de cahiers ou de livres de citations auxquels il a adjoint des "reflexions" personnelles :

"La seconde et La troisième faites a son imitation quoyque ce ne soient que) des extraits de lectures Et de reflexions d'un [?] Re(j)ent de ville (...)." (FCB 154 : AdiérazoaAvertissement f.23-25).

A cet ensemble s'ajoutent le paratexte bilingue que je viens de mentionner : Adiérazoa - Avertissement, ainsi que le poème à rimes suivies de quinze syllabes dont le titre est : Mundiren Berritzias Mosdé Etcheberri-ren Gogomenac - [Les pensées de Monsieur Etcheberri sur le Renouvellement du Monde ], sur lequel je me centrerai ultérieurement.

2. FCB 155. Sur le catalogue Omont, cet ensemble de manuscrits apparaît sous la rubrique : J. Eguiateguy Le philosophe basque. Copié sur l'éd. Francfort 1785 XIXe siècle. Suite au ms. $156 \mathrm{f} .114$. Cependant, quand on ouvre le volume qui y est répertorié on se trouve devant un titre distinct : Eguiatégui. Le philosophe basque (en souletin). 1785. Vient ensuite une page portant les mentions suivantes ler Cabier et : Filosofo buscaldun-aren Ekheïa ${ }^{7}$ Juseff Eguiatéguy Suberoan Erregent denaren obra [Ia matière du philosophe basque par Joseph Eguiatéguy, qui est régent en Soule ] suivie d'une citation latine. Au bas de la page, une adresse typographique et une date : FrancffortEn Beinat Edelman-en Moldiskidiatic MVCCLXXXV, soit "A Francfort, de l'imprimerie de Bernard Edelman, 1745". A l'évidence, il s'agit d'un avant-texte prêt à aller chez

le second de sa vieillesse. Ouvrage sont le but a été demontré que le genie de la Langue basque sous une plume telle que la sienne, est capable de la Poésie la plus élégante" (FCB 154: Avertissement f.24). Signalons que le deuxième "chapitre" des poésies d'Oihenart dont parle Eguiatéguy ne nous est pas parvenu.

6. Eguiatéguy juge qu'Oihenart est forcé d'expliquer en français les proverbes basques "a cause < du laconisme $>$ du basque qui par-dessus toutes les autres Langues se permet des sous-entendus et particulierement dans la supression des verbes. Stile qui < traduit > litteralement en françois, <rendoit> la plupart de ses pensées inintelligibles".

Eguiatéguy, en revanche, se sert surtout de recueils de citations en français pour les traduire ensuite en basque. La présence du français ayant une fonction pédagogique et pratique précise : "etre utile aux Basques qui voudroient apprendre le françois".

"Bien eloigné de l'idée de nous comparer a un tel hommelà Oihenart\}, nous n'avons donné suite a son ouvrage, qu'en faveur de la vie philosophique <pour> laquelle nous avons rapporté son ouvrage. Et comme il y à joint une traduction française nous avons crü devoir suivre son Exemple, qui pourra etre utile <ux> <Basques> qui voudroient apprendre <le françois>.

Nous avons pensé aussi que l'etendue que nous donnons aux pensées, nous dispensoient de leur donner ni application ni explication. Et qu'il seroit mieux de laisser, a un chacun cette liberté, avec celle d'assortir a son gré le moral au phisique. (...).

Nous sçavons que les Etrangers ne sont point curieux d'apprendre le Basque. ni les Basques desireux de parler les Langues Etrangeres; mais personne n'ignore que pour n'etre point duppe, il faut dans toute sorte de Commerce sçavoir la langue de ceux avec lesquels on traite, et que les praticiens avec qui les Basques commercent le plus frequament <n'Ecrivent $>$ qu'en français, il est <donc $>$ important pour eux d'apprendre cette Langue afin de ne point aller a thepitala < l'azile des mandians> par le chemin le plus court (...)" (FCB 154: Avertissement f.23-25).

De nombreux écarts existent entre l'édition Eguiatéguy et l'édition Oihenart 1657; l'édition de 1657 contient 537 proverbes et non 538 comme le ms FCB 154. En outre, Eguiatéguy change l'orthographe, introduit des néologismes empruntés à Larramendi, modifie parfois la structure syntaxique des proverbes etc.

7 - Pour Ekbeïa, Oihenart en donne la définition suivante :

"Ekaia, trauail, fa(s)herie : Item la matiere pour faire quelque ouurage, k_xa* (=) Ekhaia Edo Ekeia Le bois pour faire Vn Coffre : Guilz Ekeia Le fer pour faire Vne Clef, on dict En Labourt gaya" (Lettre adressée à Pouvreau : "Suitte de Lexplication des motz basques (...)" (FCB 8 : f.5 [25]).

Cf. également Kerejeta 1991 : 887).

* le signe diacritique placé par Oihenart sur le "u" de kuxa a, en réalité, la forme d'un "c". Faute de caractère adéquat, j'en ai placé un similaire. 
l'imprimeur. Malgré ce qui a pu être dit (Urquizu 2000 : 271 ; Orpustan 1996 : 95), et malgré l'ambiguiité de la désignation qui apparaît sur le catalogue Omont (Copié sur l'éd. Francfort 1785 XIXe sècle), aucune impression n'en a été attestée. La première édition, en une orthographe actualisée, a été réalisée par Dominique Peillen en 1983. Le manuscrit FCB 155 est entièrement rédigé en basque. Il s'agit de commentaires philosophiques développés en quarante chapitres. Le genre est connu depuis l'Antiquité ${ }^{8}$ et a donné lieu à des développements sous forme de lettres ou de traités qui ont marqué la littérature européenne médiévale et de la Renaissance (Pétrarque?, Erasme $^{10}$, Montaigne $\left.{ }^{11} . ..\right)$. Le style d'Eguiatéguy est souvent laborieux et les néologismes empruntés au grammairien et apologiste Manuel de Larramendi (Andoain 1690 - Loyola1766) ne contribuent pas à l'alléger.

Deux ultimes observations à propos du corpus FCB $155: 1$. Une liste de 42 nouveaux chapitres, qui figurent en réalité dans le fonds FCB 156, a été rajoutée à celle des 40 chapitres précédents ${ }^{12}$ qui composent la Table des Matières (Liburuaren Cekidoza) ${ }^{13} ; 2$. Eguiatéguy avait pensé faire figurer les proverbes d'Oihenart (cf. FCB 154) à la fin de ce dernier corpus 155:

Liburuaren jarraik-ian Mosdé Oỉhenarten atsotizac ta refraoc, iracourtzaler agrada siratékiela oustian, dutut jarri; (..), [J'ai placé les dits et proverbes d'Oihenart à la fin pensant que cela serait agréable au lecteur.] (FCB 155 : f.6).

3. FCB 156 : Ainsi que la mention du catalogue Omont "Suite au ms. basque 156 f.114", l'atteste, les 42 chapitres manquants dont la liste figure dans le FCB 155 se trouvent sous cette rubrique à la BNF. Deux autres corpus, qui n'ont rien à voir avec l'œuvre d'Eguiatéguy, y figurent également ${ }^{14}$.

Revenons au texte Mosdé Etcheberri-ren Mundiren Berritzias... Contrairement à l'ensemble du corpus du FCB 154, il n'a pas de version en français. Ce texte, dont la reproduction photographique de la première page avait été publiée à la page 15 de l'édition Peillen $1983^{15}$, en avait intrigué plus d'un.

En l'absence apparente de tout autre indice textuel, j'ai pensé qu'il s'avérait nécessaire de décoder, de prime abord, le titre en le lisant, non de manière segmentée (Orpustan 1996 : 97 ${ }^{16}$, mais bien comme une unité sémantique : Mundiren Berritzias Mosdé Etcheberri-ren Gogomenac - [Les pensées de Monsieur Etcheberri sur le Renouvellement du Monde $]^{17}$. On pouvait en tirer comme conséquence que : 1 . le texte n'était pas d'Eguiatéguy ; 2 . qu'Eguiatéguy inclut une information dans le titre, à savoir le nom de l'auteur de ce texte particulier : Mosdé Etcheberri. Mais de quel "Etcheberri" s'agissait-il ? Je n'en voyais qu'un qui puisse être l'auteur de ce texte : Ioannes Etcheberri de Ciboure ${ }^{18}$. Et puisque Mundiren Berritzias... était un poème eschatologique, une

8 - Pour ne citer qu'un exemple : De Vita Beata et De Providentia de Sénèque.

9-De remediid utriusque fortune [Le remède aux deux fortunes ] (1354-1366).

10 - De preparatione ad mortem [De la préparation à la mort ] (1534); Epistolae apologetica de interdicto esu carnium [Sur l'interdiction de manger de la viande ] (1522) etc.

11 - Escais (1595)

12 - Amets-Etzas [Du Rêve ], Bicitzias [De la Vie ], Demboras [Du Temps ], Sorguiñ edo Belhaguit-Etzas [Des Sorciers ou des Devins | etc.

13 - Cf. f.8.

14- On y trouve un premier corpus anonyme mais qui est de toute évidence de la main de Martin Goyhetche (A. Arcocha-Scarcia 2003) portant le titre : Cantiques. Le deuxième corpus a pour titre : (Plancas) Doctrinales en ydioma bascongado ano en 1776.

15- Sous le chapitre intitulé Berrehun urtez ixilik [Deux cents ans sous le boisseau] (Peillen 1983 : 7-22).

16- Les deux segments seraient : "Mundiren Berritzias ('Du renouvellement du monde' ou 'des mondes') et (...) Mosdé Etcbeberriren Gogomenac ('Les pensées de Monsieur d'Etcheberri')" (Orpustan 1996 : 97 ).

17 - Signalons, par ailleurs, qu'il n'y a pas de signe diacritique après le syntagme nominal Mundiren Berritzias. 18 - Auteur basque du XVII ${ }^{m e}$ siècle, réputé dans le pays pour son érudition (Iafitte, 1968: 126), sur lequel nous avons fort peu d'informations biographiques. L'un des paratextes qui figurent dans le Mamual indique 
seule ceuvre possible : le Manual Devotionezcoa ou [Manuel de Dévotion], qui comprend un vaste ensemble sur l'Apocalypse intitulé : Gviçonaren azken fiñez [Des fins dernières de l'homme] (MD 1627 ; 1669 : Livre I, 2ème partie).

C'est ainsi que j'ai observé que le texte Mundiren Berritzias..., était en réalité une adaptation $^{19}$ du poème Iujamendu ondoco munduaren statua [L'état du monde après le Jugement dernier], qui fait effectivement partie des textes sur l'Apocalypse de Ioannes Etcheberri de Ciboure. Il s'agit plus exactement du 91ème poème du chapitre III sur le Jugement dernier (MD 1627 ; 1669 : 88-89).

Le chercheur devait pouvoir accéder à la fois au texte etcheberrien tel que Joseph Eguiatéguy l'avait reçu à la fin du XVIII'̀me siècle, et au texte source tel que l'avait conçu Ioannes Etcheberri de Ciboure dans l'édition Guillaume Millanges de $1627^{20}$. J'ai pensé qu'il était nécessaire d'y ajouter une traduction en français. C'est la triple tâche à laquelle je me suis attelée en présentant une transcription annotée des deux textes et en y ajoutant une traduction commentée. Une reproduction des documents originaux accompagne l'ensemble.

\section{L'adaptation du texte source par Joseph Eguiatéguy \\ Mundiren berritzias Mosdé Etcheberri-ren Gogomenac \\ 1. Acadoykia ${ }^{21}$ acabatu datenian ${ }^{22}$ Mundian $^{23}$ \\ Ber uduri harturen du karrec ${ }^{24}$ schaoturic $^{25}$ \\ Ceruco gurutzea da gustia iraunguiren, \\ 26}

qu'il était Docteur en théologie mais on ignore de quelle université. On sait qu'il fut curé de Ciboure, on suppose qu'il y naquit puisque, comme il le rappelle dans Eliçara erabiltçeco liburua, [Le livre à utiliser pour aller à l'église], il avait été confirmé en même temps que ses trois frères par Mgr Bertrand d'Echauz (Iafitte 1968: 126).

Pierre Lafitte pense qu'il naquit dans les années 1580 : “... pour que quatre frères fussent confirmés au cours de la même cérémonie, il fallait - à moins de supposer des jumeaux - que depuis longtemps il n'y eût pas de confirmation dans la paroisse. Effectivement, après la mort de Mgr Maury, l'évêché de Bayonne était resté vacant de 1593 à 1598. On peut penser que Mgr d'Echauz a dû confirmer à Ciboure assez vite après son arrivée, dès 1598 ou en 1599. Les plus âgés des confirmands avaient sans doute environ 18 ans. Dans ce cas nous placerions la naissance de Joannes Etcheberri entre 1580 et 1586, à peu de choses près" (Lafitte 1968 : 126). Si le "feu Monsieur dEtcheberr(y)" dont Oihenart parle au passé dans la lettre qu'il adresse à Pouvreau le 30 mai 1665 à propos d'un terme de marine, est Ioannes Etcheberri de Ciboure, on peut penser qu'il est décédé avant cette date, soit bien avant la réédition de 1669 du Manual:

"Auencac les Escheles de Corde pour monter le long du mast dVn nauire feu Monsieur dEtcheberr(y) Linterprete, antemna, Mais mal a mon aduis, Car antemna se dict (maspreza) en basque". Cf. "Pour Enuoyer a Monsieur Pouureau. le 30. May 1665". (FCB 8 : f.4). Cf. également Kerejeta : 1991 : 893-894.

Trois ouvrages de Ioannes Etcheberri nous sont parvenus dont le Manual Devotionescoa [Le Manuel de Dévotion] (1627, 1669), Noelac [Noëls] (première édition en 1630 ou 1631, plusieurs rééditions, la seule nous étant parvenue étant celle de 1645) et Eliçara erabiltceco liburua [Le livre à utiliser pour aller à l'église], $(1636,1665,1666)$

19 - Le copiste Joffrion fait plusieurs erreurs lors de la transcription du texte et oublie même de copier un vers. Joseph Eguiatéguy ne semble pas avoir corrigé, comme il en a l'habitude, la copie de Joffrion qui ne connaissait manifestement pas la langue basque.

20 - Patxi Altuna avait fait paraitre une première édition du Livre I du Manual Debotionescoa en 1981 d'après l'édition de 1669. Le texte lujamendu ondoco munduaren statua y apparaissait mais il était, comme le reste des textes du Manual I présenté sous forme de distiques, alors que le texte d'origine se présente sous la forme d'une strophe compacte de 54 vers aux rimes suivies.

21 - Acadoykia : remplace Iujamendua (MD 1627; 1669). Pour Acadoykia, Dict. Michelena : "No se comprende cómo Eguiateguy e Inchauspe, que no podía conocer la obra del anterior, coinciden en sustituir ekadoi, neol. De Larramendi, por akadoi. No se trata evidentemente de un término suletino".

22 - den beçala remplace datenian (MD $1627 ; 1669$ )

23 - Mundian remplace Munduac (MD 1627; 1669). La substitution de l'ergatif singulier (munduac) par l'inessif (mundian) rend le vers de la version Eguiatéguy inintelligible.

24 - kbarrec remplace kbarrez (MD 1627 ; 1669). L'instrumental pluriel (kharrez) est remplacé par l'ergatif pluriel (karrec).

25 - schaoturic remplace le synonyme pungatuac (MD 1627 ; 1669). Pour scbaoturic, Dict. Pouvreau : "Chahutcea : nettoyer" (FCB 7 ; FCB 8). 
5. Lehen bano guehiago Eredura zazpitan

claruturen duté beré Lekhu $<$ ma $>$ natuan ${ }^{27}$,

Iguzquia Egonen da argui ${ }^{28}$ Sorthaldean $^{29}$

Ilharguia hedoy gabé date ${ }^{30}$ Sarthaldean $^{31}, \ldots$

garbi ${ }^{32}$ bersé Ceruaren charho bistagarriac

10. gao Eguinac ${ }^{33}$ ahatziren tusté beré aldiac,

Argui baizen Estaïteké ta itzalarenic

Lehen bezala issanen Ezta iduripénic

Suac Eré Ceruaren hurbilieco aozoac

Arguituren du igusgui nola vstaillacoac

15. Ceru Colore vrdinac, nola aicé ${ }^{34}$ mehiac,

Ta viaz ${ }^{35}$ diamanta nola thona ${ }^{36}$ gabiac.

Lurra beiriaren paré daïté printzanean

Ceña belaztaten ${ }^{37}$ bayta Schahuguy mahainian,

Hurian Eré Esta issanen arrain iguelduriric

20. Ez Lurrean Sagar Edo bersé frutic ${ }^{38}$ Estiric,

$\mathrm{Ta}$ aïrian Estira hegalturen schoriac

Ez hain guti Larrain Etan ibiliren abreac ${ }^{39}$,

Etenhorioc $^{40}$ onciren Lehenago inunduan ${ }^{41}$

Lurrean guizonac Egon ohiciren orduan

25. Esta beras deous issanen vstel ahal daïtenic

belhar Edo zuhaitzic Ez, Ez hetzar bicionic ${ }^{42}$

Bainan Elementuac dire izanen choil arguiac,

Ta bistac datutzanac Schahu ta garbiac

Aldapa ta mendi gorac dire ordoguiturén ${ }^{43}$,

30. ta ykayac ${ }^{44}$ Elgarren kidera bardinturen,

finean nola Cerua bayta guztiz Leonna

Lurraren bisaya hala izanen da berdina,

26 - Manque le vers suivant : Eta lebengo çuciac dire clar aguerturen (MD 1627; 1669),

27 - manatuan remplace le synonyme ordenatutan (MD 1627; 1669).

28 - argui remplace le synonyme claro (MD $1627 ; 1669)$.

29 - sorthaldean remplace le synonyme Orientean (MD 1627; 1669)

30 - date remplace berriz (MD 1627; 1669).

31 - sartbaldean remplace le synonyme Occidentean (MD 1627; 1669)

32 - Garbi remplace le synonyme Claro (MD $1627 ; 1669$ )

33 - Eguinac remplace egunec (MD 1627 ; 1669) mais la substitution de l'ergatif pluriel (egunec) par le nominatif singulier (eguinac) rend le vers incompréhensible dans la version Eguiatéguy puisque eguinac ne peut être le sujet de abatziren tusté dont le sujet est pluriel. Par ailleurs, la substitution lexicale (Eguin [faire] pour egun [jour]), change également radicalement le sens du vers d'origine.

34 - aicé [vent] remplace aire [air] (MD 1627;1669)

35-vïaz remplace vrac (MD 1627; 1669)

36 - tbona remplace le synonyme tatcha (MD 1627; 1669). Pour thona, Dict. Azkue : "Thona (S), la mancha, la tacbe. Ikhusten badu thona eztela berhatu, si viere que ella (la mancha) no se ha extendido, sil voit qu'elle (la tache) n'a pas augmenté. (Duv. Lev. XIII-53)".

37 - Pour belaztaten, Dict. Larramendi : "presentar, poner en presencia de otro, aurqueztu, betaztatu, aurrean, aitzinean ifiñi. Lat. Sistere coram aliquo".

38 - Le syntagme nominal frutic Estiric où le substantif est également mis au partitif remplace le syntagme nominal fruitu eztiric (MD $1627 ; 1669$ ) où selon la construction normale, seul l'adjectif porte la marque du partitif. le sens en est obscurci dans la version Eguiatéguy.

39-abreac remplace le synonyme bestiac (MD 1627; 1669)

40 - Eten borioc remplace Ecen borioc.

41 - inundu-an remplace munduan. Le copiste a bien respecté la transcription de l'inessif mais l'erreur de lecture, inundu pour mundu [monde] rend le vers inintelligible dans la version Eguiatéguy.

42 - bicionic, remplace bicienic (MD 1627; 1669). Une erreur d'imprimerie a dû se glisser dans l'édition MD 1627 et a dû être reconduite lors de la réimpression en 1669. La forme correcte aurait été bici(d)enic [qui vit de]. Patxi Altuna y voit la forme bizituenik (orthographe actualisée) (Etcheberri 1669 I, in édition Altuna 1981) 43 - ordoquituren remplace le synonyme planaturen (MD). Pour ordoquituren, Dict. Pouvreau : "Ordoa. Bide ordoa, bide ordoquia : chemin planier" (FCB 7; FCB 8). Dictionnaire Lhande : "ordoquitü S. : (Fx) : aplanir,". 
dohatzuac duké handi beraz ${ }^{45}$ gozomendua hain Estatu Ederrétan ikhoustés ${ }^{46}$ mundia

35. behera beondazturen ${ }^{47}$ ditu izar schahuac Elementac templa ordoki ${ }^{48}$ malkartzuac ${ }^{49}$ gaoza haoc Eguinen dire onen Errespetura, Cergatic izatu duten bici onas ardura Eten nola Erregue-Ec Soldaduen Etchéac

40. guerlatetan Eguin-Eta, Eguin Sendaguillac ${ }^{50}$ haguitz Ederraciten baituste harguiñar ${ }^{51}$, hetan Emplega detzaten manhaturic bienfunac ${ }^{52}$ hala jongoico ahalzuac ${ }^{53}$ Lur idor ta hotza Edertaturen du Noizbayt dohatzuen Egoitzua

45. Ceren Seiñalatu diren, balenties munduan Lagunec faltatu zuten arren Legué Sanduan han hara Egonen dire Ceru kuhar ${ }^{54}$ gueldiric ${ }^{55}$ (?) Azarrac, zoin beré ohatzetan jarriric. ${ }^{56}$ Minatzé-Er ${ }^{57}$ jassanen Estute ardantceric 50. Ecetordoquiec Eré alhorretan vstarric ${ }^{58}$ : Haoc lehenago onciren bicia Luzagarri guehiagoric Estaité, nihor gossé Ez Egarri.

Noizbait mundu behereco gaozac bustetcen ciren Mende honen ${ }^{59}$ joan daiteké Esta deus issaiturén.

44 - ykayac remplace le synonyme puioac (MD 1627 ; 1669). Pour ykayac, Dict. Pouvreau : "Ikaitea : monter. / Ikan naiz. j'ay monté. ikanen naiz. je monteray./Ikaitça . montée. l'action de monter" (FCB 7). Dict. Lhande 1926 : "ikai : N. (Oih. A.) côte, montée. Cf. ikhe)". Dict. Michelena : "ikai : (BN ? ap. A ; Dv (+-kh), H '-kh-). Subida".

Pour puioac: Oihenart en donne la traduction suivante : "puio || Enimence" (FCB 8 : f.7) et non "Eminence" comme le donne Kerejeta 1991 : 895. Dict. Pouvreau : "Puïo. Eminence. O". (FCB 7) ; la lettre "O" renvoie à Oihenart. Dict. Lhande 1926 : "Puio I N. (H) côte, montée, éminence, butte, colline (...)".

45 - beraz a changé de place dans la version Eguiatéguy.

46 - ikboustés remplace ikbusteaz (MD $1627 ; 1669$ )

47 - beondazturen remplace le synonyme contemplaturen (MD 1627 ; 1669). Pour beondazturen, Dict. Michelena: "beondaztu (Lar.), behondaztu. Contemplar ; ver".

48 - ordoki remplace lur (MD 1627; 1669)

49- Le vers d'origine Elementac acort, eta plano lur malkhartsuac (MD 1627 ; 1669) est bouleversé et remplacé par Elementac templa ordoki malkartzuac, le sens en devient incompréhensible dans la version Eguiatéguy.

50 - Le vers d'origine est Guerlatetan eguin eta bandi sendagailleac. (MD 1627; 1669). Ia version Eguiatéguy est rendue inintelligible par la répétition de Eguin [faire] et l'omission de l'adjectif bandi [grand].

51 - Harguinar remplace barguiñac (MD 1627;1669)

52 - Bienfunac remplace bur fuñac (MD 1627; 1669)

53 - Abalzuac (cf. Larramendi) remplace puchantac (MD 1627 ; 1669). Pour ahalzuac, Dict. Lhande : "abaltsu (A., H.) : puissant".

54 - Kubar remplace le synonyme kbechac (MD 1627; 1669). Pour kubar, Dict. Michelena : "kuinta : Queja, lamento, 'plainte' (...)". Dict. Pouvreau : "Coeita. cuita, coaita . soing, anxieté, affaire" (FCB 7 ).

Pour khechac: Dict. Lhande : "khexu: 1. soucieux, inquiet ; 4. au fig, en parlant de la mer. Itsaso kbexua, mer en courroux".

55 - La page est rognée à cet endroit.

56 - idem.

57 - Minatzé-Er remplace Mebaqueec (MD 1627 ; 1669). Pour Minatzé, Dict. Lhande 1926 : "miña S. vigne" ; "miñatze; vigne".

Pour mehaqueec: Oihenart en donne la définition suivante : "Mehaca Vn Chemin Estroit, aucunz disent quil Signiffie Vne Enimance*" (in op. cit. FCB 8: f.30). * et non : "eminance" (Kerejeta 1991: 891), cf. métathèse "m/n".

58 - Le vers d'origine était Ezta ordoquiec ere Abuztutan garbaric (MD 1627; 1669).

59- Le syntagme nominal Mende bura [littéralement : cette époque - là] (MD 1627; 1669), sujet du syntagme verbal intransitif ioan daiteque est remplacé par Mende bonen [littéralement : de cette époque-ci]. La substitution du pronom démonstratif bura [celui-là], au nominatif singulier, par le génitif singulier bonen [de celui-ci] dans la version Eguiatéguy est incorrecte et rend le sens du vers inintelligible. 

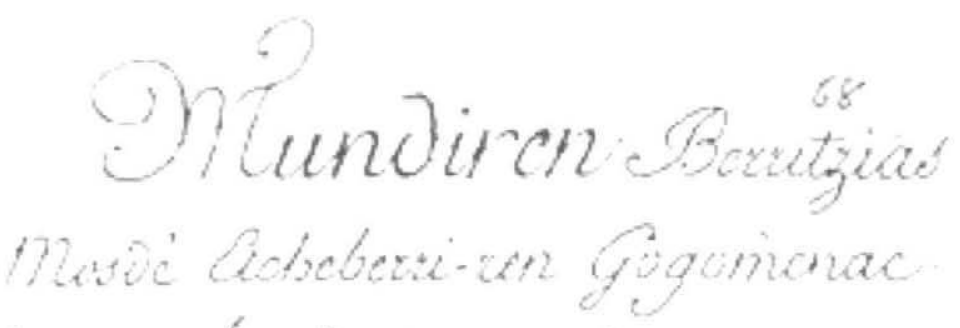

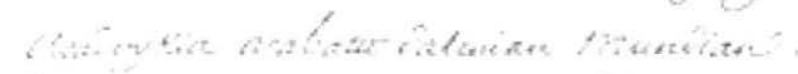

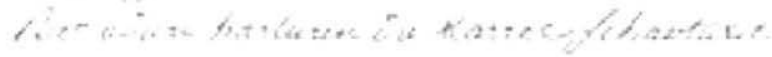

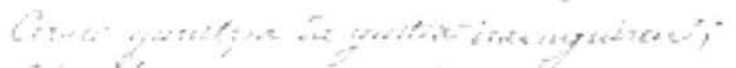

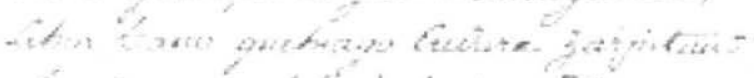

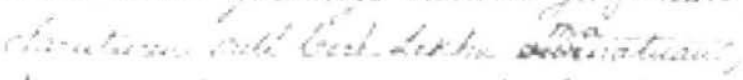

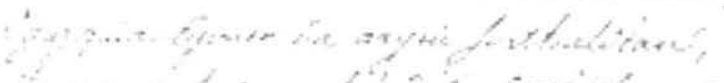

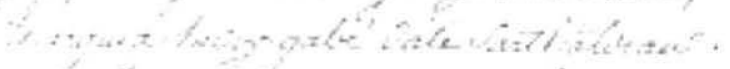

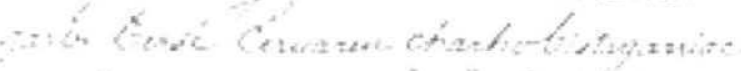

A

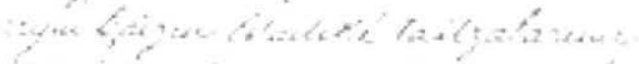

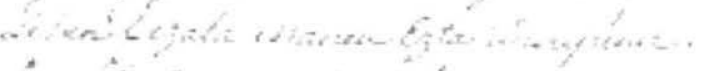

Ad

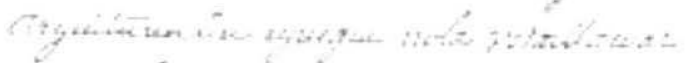

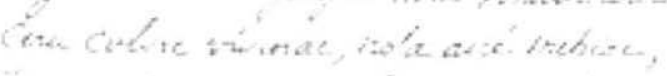

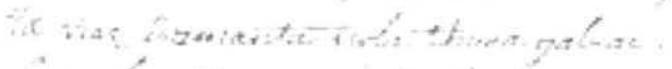

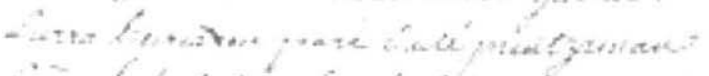

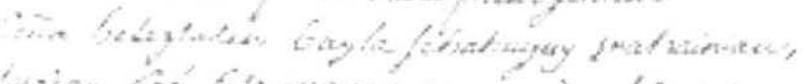

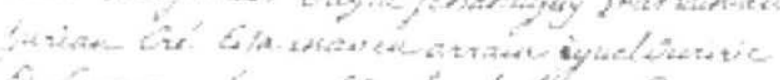

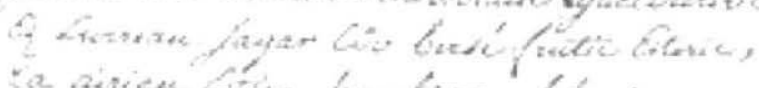

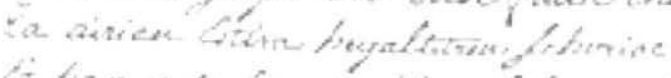

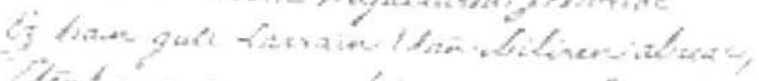

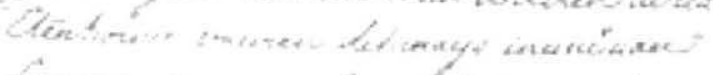

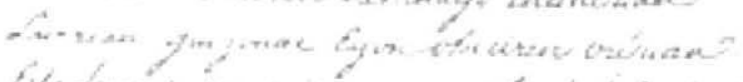

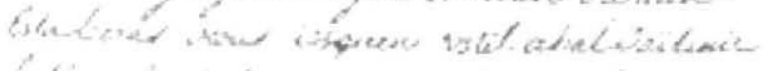

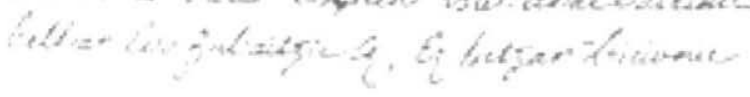

Mundiren Berritzias Mosdé Etcheberriren Gogomenac, "Réflexions de Monsieur Etcheberri sur le Renouvellement du Monde". Page droite du folio 68, recto. Ms FCB 154 de la BNF. 


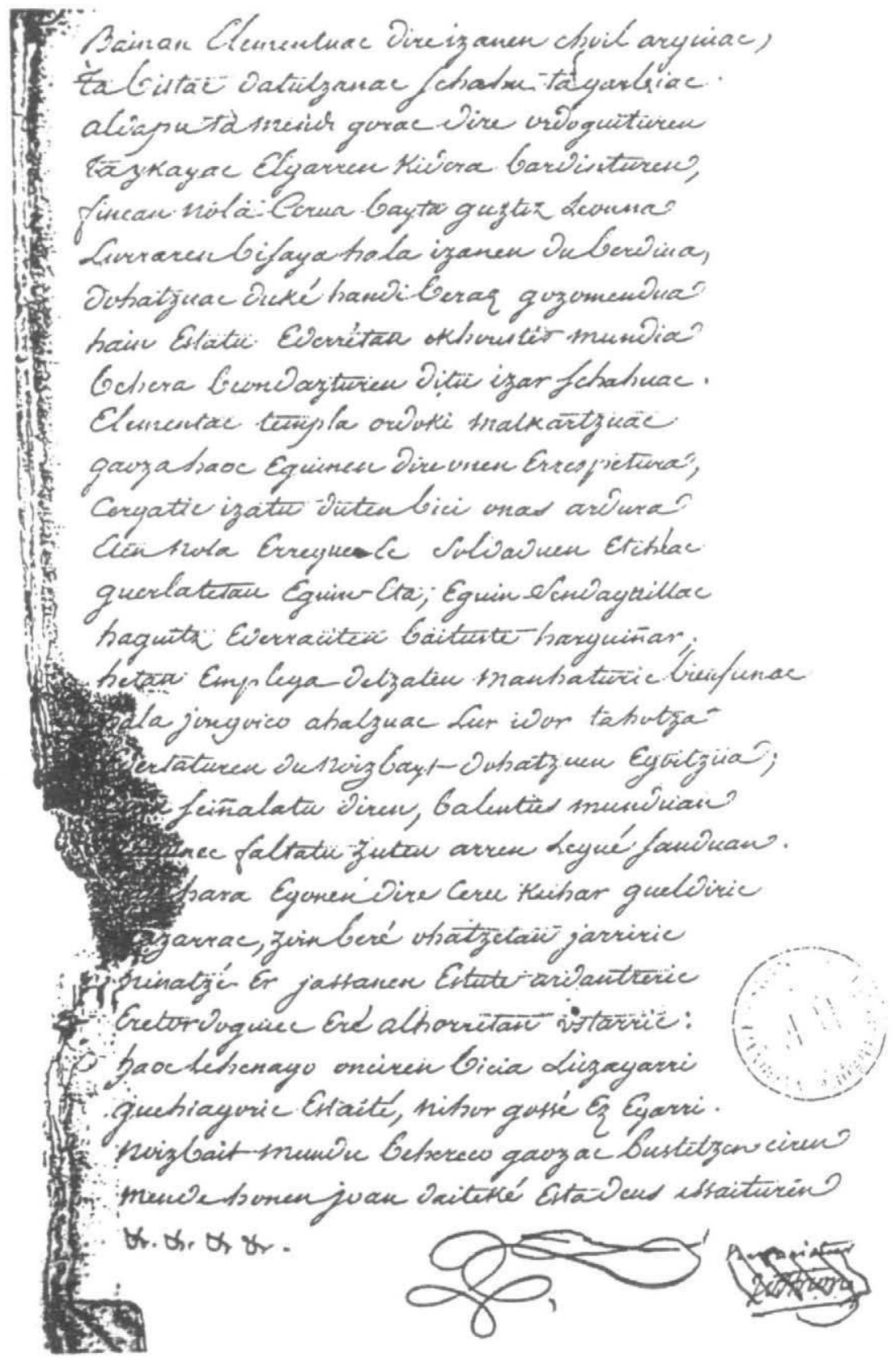

Mundiren Berritzias Mosdé Etcheberriren Gogomenac, "Réflexions de Monsieur Etcheberri sur le Renouvellement du Monde". Folio $68^{60}$, verso. Ms FCB 154 de la BNF.

60 - Le même numéro " 68 " apparaît au recto et au verso des deux feuillets, 


\section{Le texte source : Iujamendu ondoco munduaren statua de Ioannes Etcheberri de Ciboure (Manual Devotionezcoa, édition 1627 : 88-89)}

\section{Iujamendu ondoco munduaren statua}

1. IVjamendua akhabatu den beçala Munduac

Berce iduri harturen du, kharrez purgatuac, Ceruco gurutcea da guztia iraungiren, Eta lehengo çuciac dire aguerturen.

5. Lehen baño guehiago eredura çazpitan, Claraturen dute bere lekhu ordenatutan. Iguzquia egonen da claro Orientean, Illharguia hedoi gabe berriz Occidentean. Claro berce Ceruaren charho ${ }^{61}$ bistagarriac,

10. Gau egunec ahantciren tuzte bere aldiac. Argui baicen eztaiteque, eta itçalarenic, Lehen beçala içanen ezta iduripenic. Suac ere Ceruaren hurbilleco hauçoac, Arguituren du Iguzqui nola vztaillecoac.

15. Çeru ${ }^{62}$ colore vrdiñac, nola aire meheac, Eta vrac diamanta nola tatchagabeac. Lurra beiraren pare daite Princeenean Ceña presentatcen baita chahuqui mahañean. Vrean ere ezta içanen arrain iguelduriric,

20. Ez lurrean sagar, edo berce fruitu eztiric. Eta Airean eztire hegaturen choriac, Ez hain guti larrañetan ibilliren bestiac. Eçen horioc on ciren lehenago munduan, Lurrean guiçonac egon ohi ciren orduan.

25. Ezta beraz deus içanen bustel ahal daitenic, Belhar edo çuhaitcic ez, ezhetçac bicienic ${ }^{63}$. Bañan elementac dire içanen choll $^{64}$ arguiac, Eta bistac dadutçanac chahu eta garbiac. Aldapa eta mendi gorac dirade planaturen,

30. Eta puioac elkharren quidera berdinduren. Fiñean nola Cerua baita guztiz leuna, Lurraren bisaia hala içanen da berdiña. Dohatsuac beraz duque handi goçamendua, Hain estatu ederrean ikhusteaz mundua.

35. Behera contemplaturen ditu içar chahuac, Elementac acort ${ }^{65}$, eta plano lur malkhartsuac. Gauça hauc eguiñen dire onen errespetura, Cergatic içatu duten bici onaz ardura.

Eçen nola Erregueec soldaduen etcheac,

61 - "Charhoa, bougie. On sen sert par metaphore pour Les Estoiles qui éclairent La nuict". Lettre non datée adressée par Oihenart à Pouvreau intitulée : "Suitte de lexplication des motz basques demandee par Monsieur Pouureau" (FCB 8 : f.3 [f.23]). Cf. également : M.J. Kerejeta qui ajoute la date "[12 mai 166(?)]" et pense qu'il s'agit de l'année 1665. (Kerejeta ASJU 1991 : 874 et 884).

62 - Edition 1669 : Ceru.

63 - bicienic : il faut comprendre bici denic [qui vit de]. 
40. Guerlatetan eguin eta handi sendagailleac, Haguitz ederraracitcen baitituzte harguiñac, Hetan emplega detçaten manaturic bur fuñac. Hala Iongoico puchantac lur idor eta hotça, Edertaturen du noizbait dohatsuen egoitça.

45. Ceren señalatudiren valentiez munduan, Lagunec faltatu çuten arren legue Sainduan. Han hara egonen dire Çeru ${ }^{66}$ khechac gueldiric, Eta Içarrac cein bere ohatcetan ${ }^{67}$ iarriric. Mehaqueec ere iassanen eztute ardantceric ${ }^{68}$,

50. Ezta ordoquiec ere Abuztutan garbaric.

Hauc lehenago on ciren bicia luça garri, Guehiagoric eztaite nihor gosse, ez egarri.

Noizbait mundu behereco gauçac busteltcen ciren, Mende hura ioan daiteque ezta deus vsainduren.

\section{Injamend:s ondoca munduaren fatua.}

Yjamendua akhalsatu den beçala Munduac Berce iduri harturen du, kharrez purgatuac, Ceruca gurutcé da guztia iraunguiren, Eta lehengo : uciac dire clar aguerturen. Lehen ba. o guehiago credura çazpitan, Claraturen dute bere lekhu ordenatutan, Ijuzquia egonen dis claro Ori-ntean, Yllharguia hedoi gabe berriz Oecidenrean. Claroberce Ceruarea charho biftagarriac, Gau cgunec ahantciren tuzte bere aldiac. atgui baicen eztiiteque, eta itçalareniç, Zehen becala itanen ezta iduripenic. Suac ere Ceruares hurbilleco hau çoac, srguituren du Iguzqui nola vztaillecose, Çeru colore vrdínac nola aire meheak, Eta vrac diamanta nola tatchagabeac. Lurra beiraren pare daite Princeenean, Ceña prefentatceri baita chahuqui mahañean, Vrean ere czta içanen arrain iguelduriric, zz lutrean fagar, edo berce fruitu eztiric, Eta Airean eztire legaturen choriac, Ez kain guti larráietan ibilliren beftiac. Zçen horiocon cirsu lehenago munduan, "urrean guiçonase egon ohi ciren orduan. azta keju deus içi nea buftel atal daitenie 
Belharedo cuhaircic $c z$, ezhercacbicienie. Barian eleraencac dirc içauen choll arguius, Era biflac dadurçanac chahu cea garbiac. Aldapa cra mendigorac dirade planaturen, Eta puiose elkharren quidera berdinduren. Fiñean nol. Cerua baira gुuztiz lcuna, Lurraren tícia hala içanen da berdina. Dohatfuac beraz duque handi goçamendus, Hain eftacu ederean ikhufteaz nundua. Behera contemplaturen ditu içar chahtae, Elementac icort, eta plaro lur malkhartíase. Gauça haue eguiñen dire onen crérpetura, Ceroatic icaru duten bicionaz ardura. Eçen nola Erregueec foldaduen eechece. Guerlateran eguin cra handi fendagailleac. Haguirz ederraracitcen bairicuzec harguiñac, Hetun emplcga detçaten nanaturic bur furiac. Hala longoico puchantacluridorerahores, Elertatureri du noizbair doliarfuen egoirçá. Ceren fenalacudiren valencies munduan, Laggunec faleatu çuten arren legue Sainduar. Han hara gouen dire feru khechac gu=ldiric, Eta Içarrac cein berc ohatcetan iarriric, Mehaquece ere iaffanen ezcute ardanteric, Ezca ordoquiec ere Abuzcurang garbaric. Haúc lehenago on ciren bicia luça garti, Guehiagaric eztaite nihor grôle, czegurri. Noizbait muadu behereco gasçac buftelecen circn, Mende huration daiteque cata deus vianduren. monde après le Jugement]. Manual Devotionezcoa (Bordeaux 1627). 


\title{
III. Traduction du texte source ${ }^{69}:$ Iujamendu ondoco munduaren statua de Ioannes Etcheberri de Ciboure
}

\author{
L'état du monde après le Jugement dernier ${ }^{70}$ \\ Dès que le Jugement dernier ${ }^{71}$ sera accompli le Monde \\ Prendra un autre aspect ${ }^{72}$, purgés par les flammes ${ }^{73}$, \\ La croix céleste s'éteindra en entier ${ }^{74}$, \\ Et les anciennes planètes ${ }^{75}$ apparaîtront, resplendissantes. \\ Par comparaison, sept fois plus qu'avant ${ }^{76}$, \\ Elles éclaireront en leur lieu d'élection ${ }^{77}$. \\ Le soleil, éclatant, se tiendra à l'Orient \\ Tandis que la lune sera en Occident ${ }^{78}$ dans un ciel sans nuage ${ }^{79}$.
}

69 - Je remercie Bernard Oyharçabal pour les suggestions faites au sujet de la traduction.

70 - Il s'agit de la Jérusalem céleste qui vient après les deux combats eschatologiques et le Jugement des nations (Ap. 19 v11 à Ap. 20 v15)

71 - Cf. l'ensemble du Chapitre III du Manual (Bvrv III) : Iujamendua generalaz [Du Jugement général ] (1627; $1669: 60-90 ; 1981: 212-295)$

72 - Cf. Ap. 21 v5: "Alors, Celui qui siège sur le trône déclara : Voici, je fais l'univers nouveau."

73 - Il s'agit du feu purificareur qui était apparu dans le poème Azken suaren señalea [Le signe du feu ultime ] (MD $1627 ; 1669: 80-81$ ) après les présages de la fin des temps (bannière à la croix de feu, comètes dans le ciel etc, in MD $1627 ; 1669 ; 78-79)$ et qui avait brûlé tout les humains : Vste cabe barturen du Suac mumdu guztia,/Eta eztu nibor iaquinen egun baren berria./Hura da Orientetic laster auiaturen,/Eta mundu guztia du biphillduren. [Sans que nul ne s'y attende,/e monde entier prendra feu,/Nul n'aura connaissance à l'avance de ce jour,/Il [le feu] débutera rapidement en Orient,-Et rasera le monde entier.], Etcheberri écrit : ...du erreric bipillduren, qui évoque l'idée de "raser" par l'action du feu.

Cf. Ap. 16 v8-9 "Et le quatrième [Ange] répandit sa coupe sur le soleil ; alors, il lui fut donné de brûler les hommes par le feu, et les hommes furent brûlés par une chaleur torride".

74 - Sur la croix de feu, cf. précédemment le texte: Gurutce bat Ceruan aguerturen da [Une croix de feu apparaîtra dans le Ciel.] (MD 1627; 1669 : $78 ; 1981$ : 258). Il s'agit en fait d'une bannière ou d'un étendard de feu : Gurutcearen bandera, qui s'étendra lguzquia iaiquitcetic etcite lekburaño [Depuis le ponant jusqu'au couchant] et Ipharretic baice beno begoararaño ]Depuis le Nord jusqu'au vent chaud du Midi ]. Le poète ajoute : Guebiago claraturen du eçen Iguzquiac, [Elle resplendira plus que le soleil, ], (MD 1627; 1669: 78-79; 1981 : 262).

Pour la traduction de bandera, Dict. Pouvreau : "Bandera. Baniere, etendart" (FCB 7).

75 - Ia traduction de çuçia fait problème. Le terme pourrait se traduire par "torche" mais le contexte montre qu'il s'agit plutôt d'étoiles ou de planètes. Etant donné que charbo a déjà été traduit par "étoile", j'opterai pour tracłuire çuçia par "planète". Oihenart en donne la définition suivante :

"Cuçia Ceste $\mathrm{Vn}$ brandon faict dVne Souche de pin ou dautre arbre combustible qui Esclaire comme $\mathrm{Vn}$ flambeau Il se prend par metaphore pour Les planetes \& Estoiles" Iettre non datée (probablement de 1665), adressée par Oihenart à Pouvreau : "Suitte de Lexplication des motz basques demandee par Monsieur Pouureau" (FCB 8 : f.4 [f.24]), cf. également Kerejeta ASJU 1991 : 874 et 886.

Pouvreau reprend la définition dans son dictionnaire sans en préciser la provenance. (FCB 7). Ioannes Etcheberri parle ailleurs des planètes sources de lumière au Paradis : bambat planetaren claritate arguia fla clarté éclatante d'autant de planètes].

76 - Cf. Isaie 30 v26 : "Alors la lumière de la lune sera comme la lumière du soleil, / et la lumière du soleil sera sept fois plus forte, / comme la lumière de sept jours / au jour où Yahvé pansera la blessure de son peuple / et guérira la trace des coups reçus".

On peut penser qu'à la fin des temps, le mouvement des cieux ayant cessé "leur clarté augmentera" (lerner 1996 : 219). Ioannes Etcheberri a déjà consacré le poème 78 du cycle sur l'Apocalypse Cerna biguitcetic gueldituren da [Le ciel cessera de se mouvoir] à ce thème.

Le nombre sept peut évoquer également les sept planètes (désignées en latin dans les anciennes cosmographies : Lumae, Mercurii, Veneris, Solis, Martis, Iovis, Saturni), qui doivent être évoquées ici par le substantif çuciac du vers précédent au nominatif pluriel ; planètes dont l'éclat doit être plus fulgurant que dans le monde d'avant le Jugement dernier.

Au sujet de la cosmographie ancienne, cf. Lerner 1996: 203 :

"Des Babyloniens à Galilée, seuls sept astres "errants" entre le centre du monde et les étoiles fixes ont été connus des hommes : à savoir la lune et le soleil (appelés aussi "luminaires") et les cinq planètes proprement dites. Et c'est aussi autant de cieux planétaires qui seront retenus par tous les astronomes ptoléméens jusqu'au XVIème siècle".

77 - Chaque planète a son ciel, son "lieu d'élection", l'ensemble des ciels planétaires entoure concentriquement la terre. Il ne faut pas oublier que la cosmographie des peuples de l'Antiquité se retrouve dans la Bible (Champeaux/Sterckx 1981 : 56-77). Cf. par exemple le Psaume 148 :

"Louez-le [le Seigneur], soleil et lune,/louez-le, tous les astres de lumière,/ouez-le, cieux des cieux,/et les eaux 
Les autres étoiles remarquables ${ }^{80}$ du Ciel resplendiront, Les jours et les nuits oublieront leur alternance ${ }^{81}$.

Seule pourra exister la lumière ${ }^{82}$,

L'ombre ne sera en rien semblable à ce qu'elle était.

Le feu ${ }^{83}$ également, les proches voisinages du $\mathrm{Ciel}^{84}$,

Éclairera comme le soleil de juillet.

Et le ciel d'azur [éclairera] comme l'air subtil ${ }^{85}$,

Et l'eau [éclairera] comme le diamant sans tache ${ }^{86}$.

La terre, qui se présentera pure à la table ${ }^{87}$ des Princes $^{88}$,

Sera semblable au cristal ${ }^{89}$.

de dessus les cieux"! (Ps. 148 v3-4) ; le Livre de Job 22 v12 : "Dieu n'est-il pas au plus haut des cieux,/ne voit-il pas la tête des étoiles" ?; la vision de saint Paul (Ile épitre aux Corinthiens, chap, II).

78 - Cf. Isaie 60 v19-20 : "Tu n'auras plus le soleil comme lumière, le jour,la clarté de la lune sera pour toi une lumière éternelle,/et ton Dieu sera ta splendeur. Ton soleil ne se couchera plus, /et ta lune ne disparaitra plus", 79 - Les nuages entravaient non la vision de Dieu, qui par essence voit tout, mais celle des humains :

"Et parce qu'il est là-haut tu as dit : 'Que connaît Dieu ?/Peut-il juger à travers la nuée sombre ?/Les nuages sont pour lui un voile opaque/et il circule au pourtour des cieux". A la fin des temps aucune entrave n'existera plus dans le cosmos pour contempler Dieu" (Job 22 v12-14).

80 - Jentends bistagarriac, au sens de "visibles de loin", "voyants", c'est-à-dire "remarquables". Cf. également, Dict. Michelena : "bistagarri (Urt). Lo que adorna, embellece".

81 - Dieu en avait créé l'alternance le premier jour (Genèse 1 v5). L'Apocalypse en montre la fin :

"De nuit, il n'y en aura plus ; ils se passeront de lampe ou de soleil pour s'éclairer, car le Seigneur Dieu répandra sur eux sa lumière, et ils règneront pour les siècles des siècles" (Ap. 22 v5).

82 - Cf. Ap. 22 v5 et Isaie 60 v20 : "car Yahvé sera pour toi une lumière éternelle".

83 - Joannes Etcheberri décrira par ordre décroissant l'état des quatre éléments Feu, Air, Eau et Terre après le Jugement dernier (on les retrouve en latin dans les cosmographies anciennes : Ignis, Aer, Aqua, Terra). Il commence par le feu" d'en haut", substance distincte du feu terrestre selon Aristote, qui est situé au-dessus de l'air supérieur.

84 - Ceruaren burbilleco auçoac," les proches voisinages du Ciel", que l'on pourrait également traduire par les" régions proches du Ciel", désigne vraisemblablement les cercles des premières planètes, voisines effectivement du cercle igné dans les anciennes cosmographies astronomiques et théologiques.

85- En toute logique, Ioannes Etcheberri poursuit sa description en anticlimax, en passant à l'élément Air. Laction du" Ciel d'azur" est associée à celle exercée analogiquement (nola, [comme ]) par aire mebe. Ce mot composé mérite que l'on s'y arrête. En effet, il ne s'agit pas ici simplement de l'élément" air", dénommé ailleurs Aire (MD 1627 ; 1669 : 79), par Ioannes Etcheberri. Ladjectif mehe [mince] en montre la spécificité. Il semble que soit désigné ici l'air "subtil" ou "éther" dans le sens où l'utilisait Platon ("la partie pure du monde"), soit la partie suprérieure de l'élément Air.

86- Poursuivant sa descente, Ioannes Etcheberri arrive à présent au cercle Aqua/ltsassoa ou Mer supérieure qui entoure la Terre.

87 - Ia table de la Cène transfigurée, celle de la Jérusalem céleste (Hierusalen, MD 1627: 68) avec l'idée de communion entre Dieu et les élus. Par ailleurs, la forme géométrique de la table de même que laplanissement général qui s'opère sur la terre ( $\mathrm{cf}$ suite du poème de loannes Etcheberri), suggèrent que le monde nouveau est carré et non plus circulaire conformément à la forme de la Jérusalem céleste décrite dans la vision de saint Jean : "Celui qui me parlait tenait une mesure, un roseau d'or, pour mesurer la ville, ses portes et son rempart ; cette ville dessine un carré : sa longueur égale sa largeur. (...) La ville peut se passer de l'éclat du soleil et de celui de la lune, car la gloire de Dieu l'a illuminée, et l'Agneau lui tient de flambeau" (Ap.21 v15-16).

Cf. également la description du Paradis au chapitre VII du cycle sur l'Apocalypse de loannes Etcheberri (Gviçonaren azken fiñez [Des fins dernières de l'homme]) : "Lau cantoñetara eguiña da ceruric goren," (Le ciel le plus élevé a quatre côtés] ou [... s'étend sur quatre côtés] (MD 1626;1669: 133).

88 - La cour céleste de Dieu formée par le Christ "Prince des rois de la terre" (Ap. 1v5), la Vierge Marie, les Apôtres, les Docteurs de l'Eglise, les saints, les Archanges, les Anges et l'ensemble des élus inscrits dans le livre de vie et qui ne furent pas jetés dans l'étang de feu (Ap. 20 v15).

89 - Beira, Dict. Pouvreau : "Beira : verre" (FCB 7). Il me semble plus juste de traduire par "cristal" qui évoque la pierre précieuse, dans son éclat et sa transparence. La Jérusalem céleste est, en effet, resplendissante. Saint Jean raconte ainsi sa vision : "Il [l'Ange] me transporta donc en esprit sur une montagne de grande bauteur et me montra la Cité sainte qui descendait du ciel, de chez Dieu, avec en elle la gloire de Dieu. Elle resplendit telle une pierre très précieuse, comme une pierre de jaspe cristallin" (Ap. 21 v10-11).

N'oublions pas que "la pierre précieuse évoque une véritable transmutation de la matière qui, de minérale et opaque qu'elle était, devient transparente ou, pour mieux dire, devient lumière ; ce changement de l'élément le plus lourd et le plus matériel (la terre, les roches) en lumière, c'est-à-dire la quintessence de l'élément le plus léger, le plus spirituel (le feu), symbolise le passage de l'ancienne création à la nouvelle, celle de la Jérusalem céleste" (Champeaux - Sterckx 1981 : 74). 
Dans les eaux il n'y a aura pas de poisson nageurs ${ }^{90}$,

Non plus que des pommes ou d'autres fruits doux sur terre.

Et dans l'air les oiseaux ne voleront plus ${ }^{91}$,

Pas plus que les bêtes ne courront dans les plaines. ${ }^{92}$

Car ceci était bon dans le monde ancien ${ }^{93}$,

Au temps où les humains habitaient sur la terre.

Il n'y aura donc plus rien de putrescible,

$\mathrm{Ni}$ herbe ni arbre, ni aucun de ceux qui en vivent.

Mais les éléments ${ }^{94}$ ne seront que lumière,

Offerts purs et immaculés à la vue.

Les coteaux et les hautes montagnes s'aplaniront ${ }^{95}$,

Et les éminences s'égaliseront à la mesure les unes des autres.

Enfin, de même que le ciel est absolument lisse ${ }^{96}$,

Ainsi en sera-t-il de la face ${ }^{97}$ de la terre qui sera uniforme ${ }^{98}$.

Le bienheureux ressentira une jouissance intense,

En voyant la terre en un état si beau.

Il contemplera à ses pieds les étoiles pures ${ }^{99}$,

90 - Pour Iguelduric, Dict. Lhande : "igeldari L. : nageur ; igelduri : qui a la faculté de nager".

Arrivé au point central, ou culminant, de sa construction rhétorique en anticlimax, Ioannes Etcheberri se centre longuement sur l'état de la Terre d'où toute faune, flore er élément topographique a disparu lors du Jugement dernier. La mer terrestre a été annihilée (Ap. 21 v1), seul reste "le fleuve de Vie, limpide comme le cristal (...)" (Ap. 22 v1) du monde nouveau. Il faut se souvenir que saint Jean avait vu l'astre "Absinthe" tomber du ciel "sur le tiers des fleuves et sur les sources" (Ap. 8 v11). Ensuite, le deuxième et le troisième ange de Dieu avaient détruit les poissons (Ap. 16 v3-5).

91 - Ioannes Etcheberri joue sur les antithèses (future Jérusalem céleste spirituelle $\neq$ monde quotidien actuel). Son objectif consiste à déstabiliser le lecteur. Même si l'allusion n'est pas effective dans ce passage, rappelons que les oiseaux du zénith avaient été interpellés par un Ange de Dieu, puis chargés de dévorer les "nations païennes", lors du Jugement dernier:

"Puis, je vis un Ange, debout sur le soleil, crier d'une voix puissante à tous les oiseaux qui volent au zénith : Venez, ralliez le grand festin de Dieu ! Vous y avalerez chairs de rois et chairs de héros, et chairs de chevaux avec leurs cavaliers, et chairs de toutes gens, libres et esclaves, petits et grands" (Ap. 19 v17-21)!

92 - Larrañ, Dict. Pouvreau : "Larraina. plaine, vallée. place de grange. Ezcaratza. Josafateco larraina" (FCB 7). 93 - Cf. Ap. 21 v1 et 21 v3: "Puis je vis un ciel nouveau, une terre nouvelle - car le premier ciel et la première terre ont disparu, et de mer, il n'y en a plus".

Cf. également Isaie 65 v17: "Car voici que je vais créer des cieux nouveaux et une terre nouvelle,/on ne se souviendra plus du passé,/il ne reviendra plus à l'esprit".

94 - Cf. précédemment texte sur les éléments : Elementetan Señaleac, [Des signes sur les éléments ]. Les termes désignant les éléments en langue basque sont, chez Ioannes Etcheberri : Su [Feu], Aire [Air], Itsasso [Mer] et Lebor [Terre] (MD 1627; $1669: 78-79 ; 1981: 262$ ).

95 - Le septième Ange de Dieu les avait annihilés lors du Jugement dernier : "Alors toute île prit la fuite, et les montagnes disparurent" (Ap. 16 v20).

96 - La Jérusalem céleste est spirituelle, la terre est aplanie, transformée.

97 Lurraren bisaia : littéralement "le visage de la terre".

98 - Pour Berdiña, Dict. Lhande : "2. uni. Lisse". Dict. Pouvreau :"Berdina : egal" (FCB 7).

99 - Le bienheureux est soit dans le dixième, soit dans le onzième ciel ou Empyrée, créé au commencement par Dieu (Genèse 1v8).

Cf. le Livre II du Manual:

.... IElementa, eta argui Ceruetaco içarrac,/Guztiac betan nekbuzque*/sic/çre oñen azpira,/Aingueruz inguraturic non çaudecen alkira. [Les éléments, et les étoiles resplendissantes des Cieux,Je les verrais tous ensemble à vos pieds,/sous le trône où vous siégez entouré par les Anges 1. D $1627: 21 ; 1669: 19$ ). * Les deux éditions portent nekbuzque au lieu de nekbuske.

Cf. Lerner 1996 : 203 : "Un texte de la Teoria planetarium de Campanus de Novare illustre bien le problème devant lequel tout Latin faisant l'inventaire des cieux se trouvait placé. Si l'on admet qu'au-delà de la surface convexe de la neuvième sphère se trouve le ciel empyrée, séjour des bienheureux, on aura dix sphères, déclare Campanus. Mais ajoute-t-il, c'est onze qu'il faudra compter si l'on retient que le ciel cristallin (correspondant aux eaux supra-célestes de Genèse I, 7) logé sous la surface concave du ciel empyrée est distinct de la neuvième sphère. Toutefois, précise-t-il, l'astronome ne retiendra pour son usage que neuf cieux".

Le neuvième ciel ou primum mobile, puisqu'il est chargé de faire se mouvoir l'ensemble de l'univers autour de la terre, fut introduit par Ptolémée (Hypotbèses des planètes) et adopté par la suite par l'ensemble de l'Occident latin (Lerner 1996: 205). 
L'harmonie des éléments, et les terrains accidentés aplanis.

Toutes ces choses s'accompliront par respect pour les bons,

Parce qu'ils ont veillé à mener une vie bonne.

Car de même que les rois, font embellir les demeures de leurs soldats,

Suite aux grands exploits accomplis par ceux-ci dans les guerres,

En commandant aux maçons d'user à cette fin d'ingéniosité.

Ainsi Dieu tout puissant embellira un jour la terre sèche et froide,

Demeure des bienheureux ${ }^{100}$.

Parce qu'ils se sont signalés grâce à leur courage dans le monde,

Bien que leurs compagnons manquassent à la Sainte Loi ${ }^{101}$.

Ensuite, les courroux célestes s'immobiliseront ${ }^{102}$,

Et chacune des étoiles sera fixée en son nid ${ }^{103}$.

Même les terres vallonnées ne porteront plus de vigne ${ }^{104}$,

Ceci dit, les appellations des neuvième et dixième ciels peuvent varier. Le plus souvent c'est la neuvième sphère qui est désignée comme étant coelum cristallimum ou ciel aqueux, alors que la dixième est vue comme étant soit simplement le"premier mobile" et/ou également comme coelum cristallimum, théorie défendue notamment par saint Thomas : "En bonne logique, cette appellation [coelum cristallinum] aurait dû aussi englober le dixième ciel mobile dont Albert le Grand (...) paraît avoir admis l'existence (...). Cela porterait à deux le nombre des cieux dits aqueux ou cristallins, comme saint Thomas le suggérait (...). La question ne semble pas cependant avoir jamais fait l'objet d'une attention spéciale, et l'on constate que les diagrammes du monde avec dix cieux mobiles désigneront le plus souvent comme cristallimum (ou aqueum) la seule neuvième sphère députée au mouvement de trépidation, la dixième, celle du mouvement diurne, se voyant appeler sans autre précision Primum Mobile. Parmi les rares cas où apparaît l'indication de deux cieux cristallins, nous mentionnerons le diagramme (...) illustrant la Summa pbilosopbica quadripartita d'Eustache de saint Paul, un manuel à succès publié une première fois à Paris en 1609" (Lerner 1996: 214).

Cf.Giovanni Tolosanni in Compendio di spbaera et machina del mondo muovamente composto (1514) :

La decima \& la nona esser cbristalline

diaphane come acque transparente

sopra loctava ciaschuma exser vicina

E son chiamate $\mathcal{G}$ decte acque eminente (...) (cité par Lerner 1996: 364)

Les Bienheureux dont parle le texte de Ioannes Etcheberri, sont situés dans un Empyrée qui semble surplomber directement le huitième cercle des étoiles fixes. A moins que la présence d'un neuvième (et/ou d'un dixième) cercle ne soit implicite. Pour les théologiens le ciel cristallin est, en effet, "uniformément diaphane, à la différence notable de la sphère étoilée et dans une moindre mesure des sphères porteuses d'une planète, qui sont localement brillantes (lucida) (...)" (Lerner 1996: 214). Les Bienheureux peuvent ainsi directement admirer les étoiles à leurs pieds, "vers le bas" (Bebera contemplaturen). Ce ciel cristallin est celui de Genèse 1-7: "Dieu fit le firmament, qui sépara les eaux qui sont sous le firmament d'avec les eaux qui sont au-dessus du firmament, et Dieu appela le firmament "ciel".

100 - Ies Bienheureux vivront dans un monde transformé où la terre détruite entièrement lors du Jugement dernier (lur idor eta botça, [terre sèche et froide]), sera offerte, métamorphosée, aux Bienheureux qui circuleront librement entre tous les cercles de l'univers rendu à l'unité divine première.

Les adjectifs idor [sèche] et botça [froide] peuvent renvoyer aux théories des penseurs de l'Antiquité grecque comme Théon de Smyrne pour qui la Terre est "froide" par rapport au soleil qui est un astre "chaud".

101 - Les "compagnons" (lagunec) qui ont manqué à "la Sainte Loi" (Legué Sanduan) ont été jugés coupables lors du "Jugement des nations" et jetés dans "l'étang de feu" :

"Et la mer rendit les morts qu'elle gardait, la Mort et l'Hadès rendirent les morts qu'ils gardaient, et chacun fut jugé selon ses ceuvres. Alors la Mort et l'Hadès furent jetés dans l'étang de feu - c'est la seconde mort, cet étang de feu- et celui qui ne se trouva pas inscrit dans le livre de vie, on le jeta dans l'étang de feu" (Ap. 13 v15).

102 - Dans la Jérusalem céleste, I'harmonie sera totale entre Dieu et ses créatures, Dieu n’aura donc plus de raison s'être courroucé :

"Voici la demeure de Dieu avec les hommes. $\mathrm{I}$ aura sa demeure avec eux ; ils seront son peuple, et lui, Dieuavec-eux, sera leur Dieu. Il essuiera toute larme de leurs yeux : de mort, il n'y en aura plus ; de pleur, de cri, de peine, il n'y en aura plus, car l'ancien monde s'en est allé' (Ap. 21 v3-4).

103 - Il doit s'agir du huitième ciel porteur des étoiles fixes, ajouté par les astronomes médiévaux. Sur la fixation des étoiles diverses théories ont été émises depuis les penseurs grecs, en rapport avec les différentes idées que l'on a pu se faire au sujet de la solidité des sphères et en particulier de celle où figurent les étoiles "fixes".

Tout est à nouveau dans l'ordre, dans le monde renouvelé qui vient après la fin des temps. Les étoiles sont "fixées", les planètes à leur place etc.

104 - Cf. également, Dict. Lhande : Mebakek ere eztute iasanen ardantzarik J. Etch. -H.) "les vignes ne produiront pas non plus de vendanges" (1926: 54). 
Ni les plaines de gerbes en août.

Ces choses étaient auparavant bénéfiques pour prolonger la vie,

Désormais nul n'aura plus ni faim ni soif.

Il fut un temps où les choses d'ici-bas se décomposaient ${ }^{105}$,

Ce temps-là s'en ira ${ }^{106}$, et rien de nauséabond ${ }^{107}$ n'existera.

\section{Bibliografia}

- ARCOCHA - SCARCIA, A. 2003 : "Manuscrits relatifs aux fables de La Fontaine et de Florian traduites et adaptées du français au basque labourdin par Martin Goyhetche (1791-1859)". Lapurdum n7, Bayonne. 25-83.

- ATUTXA, Isaac, 2001 : "Joannes Etxeberriren Manual devotionezcoa-z", Lapurdum VI, Bayonne.

- AZKUE, R. M. 1905 [1984] : Diccionario vasco-español-francés, Dictionnaire Basque - Espagnol - Français. Euskaltzaindia, Bilbao.

- BIBLE DE JERUSALEM (la), [1975] : Editions du Cerf, Paris.

- BILBAO, G. 1992 : "Pouvreauren Hiztegi Laukoitza" , ASJU, Saint Sébastien. 341-389.

- BRUNO, G. [1995] : De l'infini, de l'univers et des mondes. Texte bilingue italien / français, transcrit selon l'editio princeps Charlewood J., Londres 1584, (fausse adresse typographique de Venise). Edition : Aquilecchia G., trad. : Cavaillé P., Les Belles Lettres, Paris.

- CHAMPEAUX, G. (de), STERCKS, S. (dom), 1981 : Le monde des symboles, Editions Zodiaque, La Pierre-qui-Vire.

- EGIATEGI, J. [1983] : Leben Liburia edo Filosofo buskaldunaren ekbeia (1785). Edition : Peillen T., collection : Euskararen lekukoak, Euskaltzaindia, Bilbao.

- ERASME, [1992] : Eloge de la Folie, Adages, Colloques, Réflexions sur l'Art, l'Education, la Religion, la Guerre, la Philosophie, Correspondance. Collection Bouquins, Robert Laffont éditions, Paris.

- ETCHEBERRI, I. 1627 : Manval Devotionezcoa edo ezperen, oren oro escuetan erabilltçeco liburutchoa. Escarazco versu tan eguiña, eta guztia bi partetan bereçia. Bordelen Gvillen Millanges, Erregueren Imprimat çaillearenean. M. DC. XXVII.

- ETCHEBERRI, I. 1669 : Manval Devotionezcoa edo ezperen, oren oro escuetan errabilltçeco liburutchoa. Escarazco versutan eguiña, eta guztia bi partetan berecia. Bordelen I. Mongiron. Millanges, Erregueren Imprimatçaillearenean. Edition facsimile de cette seconde édition, Hordago, Saint-Sébastien, 1978.

- ETCHEBERRI, I. 1669 [1981] : Manual Devotionezcoa (Leben zatia) (Edizio kritikoa) Patxi Altunak paratua. Edition critique de la première partie du Manual Debotionezcoa (éd. 1669) établie par P. Altuna, Ediciones Mensajero, Bilbao.

- HARITSCHELHAR, J. 1992 : "Parémiologie et mnémotechnie. L'exemple basque". Bulletin du Musée Basque n¹33, 49-66, Bayonne.

— KEREJETA, M.J. 1991 : "Oihenart Silvain Pouvreauren hiztegian". ASJU, Donostia - San Sebastián. 865-899.

— LAFITTE, P. 1968 : "Copiage bio-bibliographique autour du poète 'Jean d'Etcheverry'”, Gure Herria, 40ème année, Bayonne. 123-128.

105 - Le processus de la décomposition suppose la mort. Ce n'est plus le cas de la Jérusalem céleste (cf. Ap. 21 v4 ). 106 - Cf. Isaïe 65 v16-17 : "(...) on oubliera les angoisses anciennes, / elles auront disparu de mes yeux. (...) on ne se souviendra plus du passé/il ne reviendra plus à l'esprit".

107 - La mauvaise odeur est signe de désordre, elle est associée au monde infernal. Au Paradis, séjour des Bienheureux, il n'y a que de "bonnes" odeurs. 
- LAFITTE, P. 1941 : Le basque et la littérature d'expression basque en Labourd, Basse-Navarre et Soule. Conférence donnée au Musée Basque de Bayonne le 3 avril 1941. Collection "Aintzina", Bayonne.

- LAFITTE, P. 1931 : Eskualdunen Loretegia. XVI garren mendetik bunako liburuetarik bildua. Lehen zathia (1645-1800). Lasserre liburutegian salgei, Bayonne.

- LARRAMENDI, M. (Padre de) 1745 : Diccionario trilingue del castellano, bascuence, y latin. Tome I et II, Edition Riesgo y Montero, Saint Sébastien.

- LERNER, M.-P. 1996 : Le monde des sphères. Tome I, Genèse et triomphe d'une représentation cosmique, Les Belles Lettres, Paris.

- LHANDE, P. 1926 : Dictionnaire Basque-Français. Gabriel Beauchesne éditeur, Paris. Littré, dictionnaire de la langue française, 1962 : Bergame.

- MICHELENA, K. 1987-2004 : Diccionario general vasco - Orotariko euskal biztegia. Real Academia de ma Lengua Vasca - Euskaltzaindia, Bilbao.

- MONTAIGNE, M. (de) [2002] : Les Essais, Livres I et II. Reproduction de l'édition Marie de Gournay de 1595, Le Livre de Poche, Paris.

— OIHENART, A. [1992] : Proverbes et Poésies basques. Edition : Orpustan, Izpegi, Saint-Etienne-de-Baïgorry.

- OIHENART, A. [2003] : Euskal atsotitzak eta neurtitzak-Proverbes et Poésies basques-Proverbios y poesías vascas. Edition de la Préface, des Proverbes et de leurs notes : Jose Antonio Mujika ; édition des Poésies et de leurs notes : Patxi Altuna, Iker 15, Euskaltzaindia, Bilbao.

— OIHENART, d', 1657 : Les proverbes basques recueillis par le Sr d'Oibenart, plus les poésies basques du même auteur. Paris. [fac-simile]

- OIHENART, A. (d') 1638/1656 [1992]. Notitia Utriusque Vasconiae, tum Ibericae, tum Aquitanicae, qua praeter situm regionis et alia scitu digna, Navarrae Regum, Gasconiae Principium, caeterarumque, iniis, insignium vetustae et dignitate familiarum stemmata ex probatis Autboribus et vetustis monumentis exbibentur. Accedunt Catalogi Pontificum Vasconiae Aquitanicae, bactenus editis pleniores. [Noticia de las dos Vasconias, la Ibérica y la Aquitana, en la que se describen, además de la situación de la región y otras cosas dignas de conocerse, la genealogía de los reyes de Navarra, de los principes de Gascuña, y otras familias ilustres por su antigüedad y dignidad, conforme se hallan en los autores antguos : Se añaden los catálogos de los Prelados de la Vasconia Aquitana, más completos que los publicados basta abora... Reproduction facsimile de la deuxième edition, Paris 1656, étude préliminaire de R. Ciervide, Eusko Legebiltzarra - Parlamento Vasco, Vitoria, 1992.

— ORPUSTAN, J.-B. 1999. "Un type de "paratexte ' dans la littérature basque de la première moitié du XVII e siècle : les dédicaces", Actes du colloque de Bayonne" Les lettres basques au temps d'Axular", Lapurdum n4, Bayonne. 9-37.

- ORPUSTAN, J.-B. 1996 : Précis d'bistoire littéraire basque 1545-1950. Cinq siècles de littérature en euskara. Izpegi éditions, Saint-Etienne-de-Baigorry.

- OYHARÇABAL, B. 2002. "De l'usage de l'étrangeté syntaxique : les structures agrammaticales dans le versification basque du 17 ème siècle." In Erramu boneta : Festschrift for Rudolf P. G. de Rijk, Xabier Artiagoitia, Patxi Goenaga, Joseba A. Lakarra (arg. / eds.), ASJU, Universidad del País Vasco - Euskal Herriko Unibertsitatea, Bilbao.

- OYHARÇABAL, B. 2001. "Statut et évolution des lettres basques durant les XVIIe et XVIII" siècles", Lapurdum $n^{\circ}$ 6, Bayonne, 219-189. 
- OYHARÇABAL, B. 1999. "Les prologues auctoriaux des ouvrages basques des XVIe et XVIIe siècles", Lapurdum n 4 Numéro spécial, Bayonne, 39-93.

— PEILLEN, T. 1994 : "Oihenarteren eragina Egiategirengan (Oihenarteren itzala Egiategiren obran)". Iker 8, édition monographique sur Oihenart, Euskaltzaindia, Bilbao, 467-476.

- PETRARQUE, [2002] : Les remèdes aux deux fortunes, Vol. I et II. Editions Jérôme Millon, Grenoble.

- SENEQUE, [2002] : La Vie heureuse. La providence. Les Belles Lettres, édition bilingue Latin-Français, Paris.

- URQUIZU, P. 2000 : Historia de la literatura vasca. U.N.E.D., Madrid.

\section{Manuscrits de la BNF consultés :}

OMONT, H. 1890 : Catalogue des manuscrits celtiques et basques :

- $\mathrm{ms} \mathrm{n}^{\circ} 7$ : Dictionnaire basque-français, par Silvain Pouvreau, prêtre du diocèse de Bourges. XVIIe siècle (...);

- $\mathrm{ms} \mathrm{n}^{\circ} 8$ : Le même dictionnaire. Incomplet du commencement (...);

- $m s n^{\circ} 154$ : Sentences et proverbes d'Oybenart.

- $\mathrm{ms} \mathrm{n}^{\circ} 155$ : J. Eguiateguy Le philosophe basque. Copié sur l'éd. Francfort 1785 $X I X^{e}$ siècle.

- $\mathrm{ms} \mathrm{n}^{\circ} 156$ (FCB 156) : Suite au ms. $156 f .114$ [suite du corpus Eguiatéguy FCB 155]. 